\title{
Analysis and Optimal Condition of the Rear-Sound-Aided Control Source in Active Noise Control
}

\author{
Karel Kreuter ${ }^{1}$ and Yasuhide Kobayashi ${ }^{2}$ \\ ${ }^{1}$ Department of Electrical Engineering, Technical University of Darmstadt, 64283 Darmstadt, Germany \\ ${ }^{2}$ Department of Mechanical Engineering, Faculty of Engineering, Nagaoka University of Technology, Nagaoka, Niigata 940-2188, Japan
}

Correspondence should be addressed to Yasuhide Kobayashi, kobayasi@vos.nagaokaut.ac.jp

Received 14 March 2011; Revised 3 May 2011; Accepted 18 May 2011

Academic Editor: Arnaud Deraemaeker

Copyright ( $\odot 2011$ K. Kreuter and Y. Kobayashi. This is an open access article distributed under the Creative Commons Attribution License, which permits unrestricted use, distribution, and reproduction in any medium, provided the original work is properly cited.

An active noise control scenario of simple ducts is considered. The previously suggested technique of using an single loudspeaker and its rear sound to cancel the upstream sound is further examined and compared to the bidirectional solution in order to give theoretical proof of its advantage. Firstly, a model with a new approach for taking damping effects into account is derived based on the electrical transmission line theory. By comparison with the old model, the new approach is validated, and occurring differences are discussed. Moreover, a numerical application with the consideration of damping is implemented for confirmation. The influence of the rear sound strength on the feedback-path system is investigated, and the optimal condition is determined. Finally, it is proven that the proposed source has an advantage of an extended phase lag and a time delay in the feedback-path system by both frequency-response analysis and numerical calculation of the time response.

\section{Introduction}

In the recent past the concept of active noise control (ANC) has arrest more attention. Due to the falling prices for digital signal processors (DSPs), ANC applications become more popular everyday. The Swinbanks' (unidirectional) source [1] has frequently been applied to control noise scenarios to show its advantages over a standard bidirectional case with one control source. The implementation has shown to be of lower cost and at the same time better control performance. This has been confirmed by experiments in both adaptive and robust control setups $[2,3]$. A welcome side effect was a lower controller gain and driving signals for the used loudspeakers [3, 4], which enables the usage of lower power loudspeakers. The advantage of the Swinbanks' source has been theoretically proven for a practical setting on the duct length and loudspeaker locations $[5,6]$.

The intention of the Swinbanks' source is cancelling of the upstream sound, which is emitted by the downstream loudspeaker. In order to accomplish this, the upstream source is driven with the same amplitude as the downstream loudspeaker but opposite phase and proper time delay. Thus, the upstream sound at the mount of the upstream source is eliminated. The advantage in the control performance can be explained by the extension of the time period by which the sound emitted by the control source travels to the reference microphone. It has been proven that the Swinbanks' source introduces an additional time delay corresponding with twice the distance between control source and duct end $[5,6]$. Previous research pointed out on empirical basis that the closed-loop performance is improved when the actuator (control source) and the sensor (reference microphone) are of further distance $[7,8]$.

In addition to the Swinbanks' source, the rear-soundaided source has been proposed to achieve similar advantage of Swinbanks' source by using one loudspeaker [9]: the source is composed of a loudspeaker and a subduct which connects the rear side of the loudspeaker to an upstream junction in the main duct in order to attenuate the upstream sound by interfering the rear sound as shown in Figure 1 . Experiments demonstrated that the proposed source has an advantage. It achieves a lower amplitude of error microphone signal by a smaller amplitude of control input over the conventional bidirectional source. 


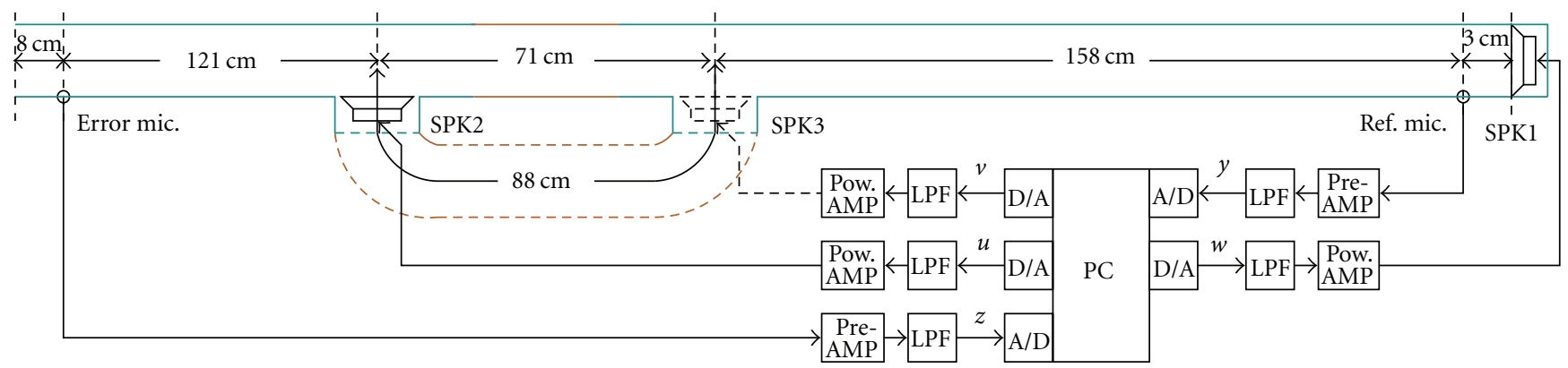

Figure 1: Experimental apparatus used in [9].

However, the following problems remain for the proposed source.

(i) The first principle model derived in [9] has not been well consistent with the experimental result of the frequency response.

(ii) No advantage of the proposed source has been shown theoretically: the additional time delay in the feedback-path transfer function has not been analyzed.

In this paper, we concentrate on the second problem, because the novel contribution of showing an advantage in a theoretical way is also necessary to solve for the first problem in the future.

In order to show the advantage of the proposed source, feasibility of the additional phase lag in the frequency response and the additional time delay in the time response are both investigated in this paper based on the frequency response analysis by the first principle model and the time-response simulation by the numerical calculation. In addition, the consistency between the frequency response analysis and the time response simulation will be shown to validate an assumption involved in the simulation model due to the junction of the proposed source, which is not necessary for more simpler structures of the conventional sources. To show consistency, the frequency response is numerically calculated by injecting a sinusoidal sound, where the damped wave equation is used to consider energy dissipation. Herewith, the steady-state response does not diverge for resonance frequencies.

This paper is outlined as follows. Firstly, the first principle model [9] is derived based on the damped wave equation which later enables comparison of the frequency response of the first principle model with the numerical simulation. In Section 3, the damped wave equation will be directly implemented in a numerical simulation by using finite differences. We will demonstrate that the frequency response calculated by numerical implementation is consistent with the one given by the first principle model. Thus, the model will be available to examine both the additional phase lag and time delay in the feedback-path transfer function. Finally, the optimal condition achieving the longest time delay on the feedback-path transfer function will be given based on the first principle model in Section 4. The condition will be used in numerical simulations to directly visualize the additional time delay.

\section{First Principle Model}

In this section, the damped wave equation is used to derive frequency response functions in order to obtain a finite amplitude response at resonance frequencies. The damped wave equation is the wave equation with an additional term of the first partial derivative of pressure in time (see, e.g., [10]). The differences to the existing result of [9] will be explained, since the existing result is based on the (undamped) wave equation. Note that the derivation process of the damped wave equation will be described in this section, since the intermediate equations ((1) and (2) below) are necessary to exploit the existing result of the transmission line theory.

The damped wave equation can be derived by two physical laws, the conservation of mass (1) and momentum (2). The acoustic velocity is given by $u, p$ represents the pressure. Note that an additional term $d u$ is included in the conservation of momentum to take friction effects into account, where $d$ is a damping coefficient,

$$
\begin{gathered}
\frac{1}{c_{0}^{2}} \frac{\partial p}{\partial t}+\rho_{0} \frac{\partial u}{\partial x}=0 \\
\rho_{0} \frac{\partial u}{\partial t}+\frac{\partial p}{\partial x}+d u=0 .
\end{gathered}
$$

If the damping is neglected, the two equations are well known in acoustic literature (see, e.g., [11]) and are used to derive the wave equation without consideration of damping. However, to the best of our knowledge, (2) with $d \neq 0$ has not been used to derive a physical model in the field of active noise control. Usually, the damped wave equation (3) is solved without considering damping. The resulting undamped solution is used to approximate damping effect by modifying the wave number $k:=\omega / c_{0}$ (real number) to $k:=\left(\omega / c_{0}\right)+j \alpha$ (complex number) [12], which technique has also been used in [9]. The damped wave equation can be obtained by the derivation of (1) with respect to $t$ and (2) with respect to $x$ and combining both

$$
\frac{1}{c_{0}^{2}} \frac{\partial^{2} p}{\partial t^{2}}+\frac{d}{\rho_{0} c_{0}^{2}} \frac{\partial p}{\partial t}=\frac{\partial^{2} p}{\partial x^{2}} .
$$


On the other hand, more general partial differential equations and the solution with damping consideration are common in the transmission line theory. It is well known for instantaneous voltage $v(x, t)$ and current $i(x, t)$ at a location $x$ on a lossy transmission line that the following relations hold:

$$
\frac{\partial v}{\partial x}=-R i-L i \frac{\partial i}{\partial t}, \quad \frac{\partial i}{\partial x}=-G v-C \frac{\partial v}{\partial t},
$$

where $R, L, C$, and $G$ are resistance, inductance, capacitance, and conductance per unit length of the line. One can verify that (4) equals (1) and (2) when using the following relations:

$$
\begin{gathered}
v(x, t)=p(x, t), \quad i(x, t)=u(x, t), \quad R:=d, \\
L:=\rho_{0}, \quad G:=0, \quad C:=\frac{1}{\rho_{0} c_{0}^{2}} .
\end{gathered}
$$

For harmonic signals

$$
v(x, t):=\operatorname{Re}\left\{V(x) e^{j \omega t}\right\}, \quad i(x, t):=\operatorname{Re}\left\{I(x) e^{j \omega t}\right\},
$$

the partial differential equation of transmission line theory can be rewritten as

$$
\frac{d V}{d x}=-Z I, \quad \frac{d I}{d x}=-Y V,
$$

where $Z:=R+j \omega L$ and $Y:=G+j \omega C$ are impedance and admittance per unit length. The general solution of (7) is known as

$$
V=A e^{-\gamma x}+B e^{\gamma x}, \quad I=\sqrt{\frac{Y}{Z}}\left(A e^{-\gamma x}+B e^{\gamma x}\right),
$$

where $A$ and $B$ are complex-valued integral constants. $\gamma$ is given by

$$
\begin{aligned}
& \gamma:=\sqrt{Z Y}:=\alpha+j \beta \\
& \alpha:=\sqrt{\frac{1}{2} \sqrt{R^{2}+\omega^{2} L^{2}} \sqrt{G^{2}+\omega^{2} C^{2}}-\left(\omega^{2} L C-R G\right)} \\
& \beta:=\sqrt{\frac{1}{2} \sqrt{R^{2}+\omega^{2} L^{2}} \sqrt{G^{2}+\omega^{2} C^{2}}+\left(\omega^{2} L C-R G\right)} .
\end{aligned}
$$

Lemma 1. In transmission line, suppose that $v(x, t)$ and $i(x, t)$ are in steady state with the relations given in (6). Then, $V(x)$ and $I(x)$ at arbitrary position $x$ is related with the values at origin, $V(0)$ and $I(0)$, by

$$
\left[\begin{array}{l}
V(x) \\
I(x)
\end{array}\right]=\left[\begin{array}{cc}
\cosh (\gamma x) & -Z_{0} \sinh (\gamma x) \\
-\frac{1}{Z_{0}} \sinh (\gamma x) & \cosh (\gamma x)
\end{array}\right]\left[\begin{array}{c}
V(0) \\
I(0)
\end{array}\right],
$$

where $Z_{0}:=\sqrt{Z / Y}$ is the characteristic impedance of the transmission line.

Proof. By substituting $x=0$ into (8), $A$ and $B$ are written in $V(0)$ and $I(0)$. Then, the elimination of the constants $A$ and $B$ leads to (10).
Likewise, a similar lemma holds for the sound propagation in a straight duct.

Lemma 2. Suppose that $p(x, t)$ and $u(x, t)$ are in steady state with the relation given as

$$
p(x, t):=\operatorname{Re}\left\{p(x) e^{j \omega t}\right\}, \quad u(x, t):=\operatorname{Re}\left\{u(x) e^{j \omega t}\right\} .
$$

Then, $p(x)$ and $u(x)$ at arbitrary position $x$ is related with the values at origin, $p(0)$ and $u(0)$, by

$$
\left[\begin{array}{l}
p(x) \\
u(x)
\end{array}\right]=\left[\begin{array}{cc}
\cosh (\gamma x) & -Z_{0} \sinh (\gamma x) \\
-\frac{1}{Z_{0}} \sinh (\gamma x) & \cosh (\gamma x)
\end{array}\right]\left[\begin{array}{l}
p(0) \\
u(0)
\end{array}\right],
$$

$$
\begin{gathered}
Z_{0}:=\rho_{0} c_{0} \sqrt{1+\frac{d}{j \omega \rho_{0}}, \quad \gamma:=\alpha+j \beta,} \\
\alpha:=\frac{1}{\sqrt{2} c_{0}} \sqrt{\omega \sqrt{\omega^{2}+\frac{d^{2}}{\rho_{0}^{2}}}-\omega^{2},} \\
\beta:=\frac{1}{\sqrt{2} c_{0}} \sqrt{\omega \sqrt{\omega^{2}+\frac{d^{2}}{\rho_{0}^{2}}}+\omega^{2},}
\end{gathered}
$$

where $Z_{0}$ is called the acoustic characteristic impedance of the duct.

Proof. It is trivial from Lemma 1 and the relations (5).

The next lemma is used to derive first principle model so that series connections of ducts are simplified in the derivation process, which is an extended result to the undamped case in [9].

Lemma 3. Define a matrix $T(l)$ with arbitrary complex numbers $\gamma$ and $Z_{0}$ by

$$
T(l):=\left[\begin{array}{cc}
\cosh (\gamma l) & -Z_{0} \sinh (\gamma l) \\
-\frac{1}{Z_{0}} \sinh (\gamma l) & \cosh (\gamma l)
\end{array}\right] .
$$

Then, for any positive real number $x$ and $y$, the following holds:

$$
T(x) T(y)=T(x+y) .
$$

Proof. It can be verified by multiplication and application of the theorems for sinh and cosh.

The relationship of pressure $p$ and acoustic velocity $u$ between different points in space will serve as the basis for deriving a unifying transfer function which includes the proposed control source. We consider the duct system in Figure 2 which is the same as in [9], where SPK1 is a noise source, SPK2 and SPK3 are used as a control source, controller is connected between $y$ and $u$ to drive the control source based on the information of the reference microphone 


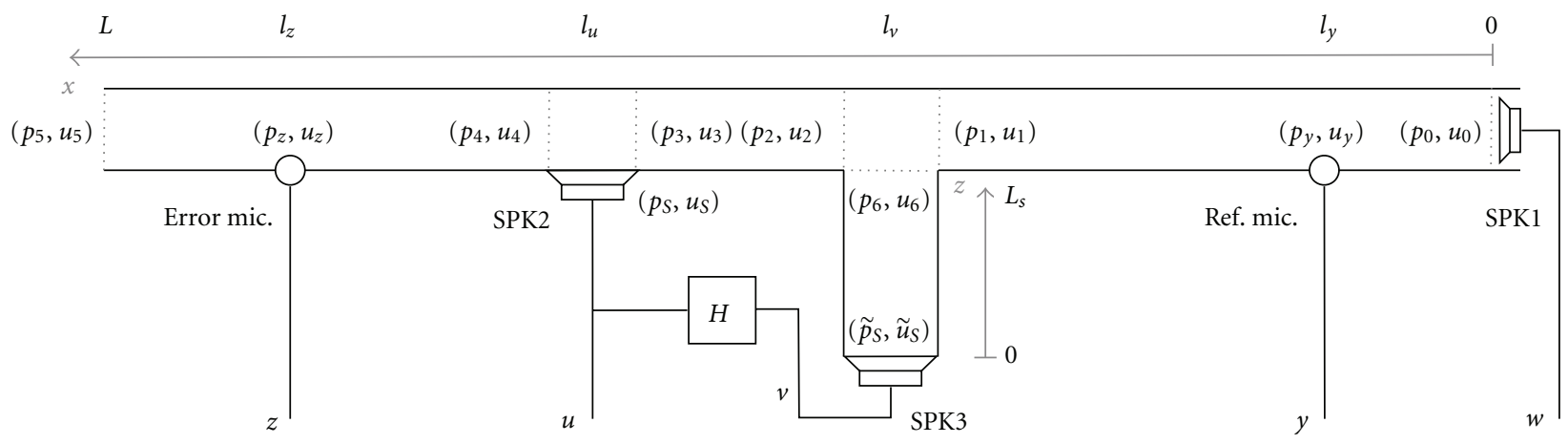

FIGURE 2: Theoretical apparatus.

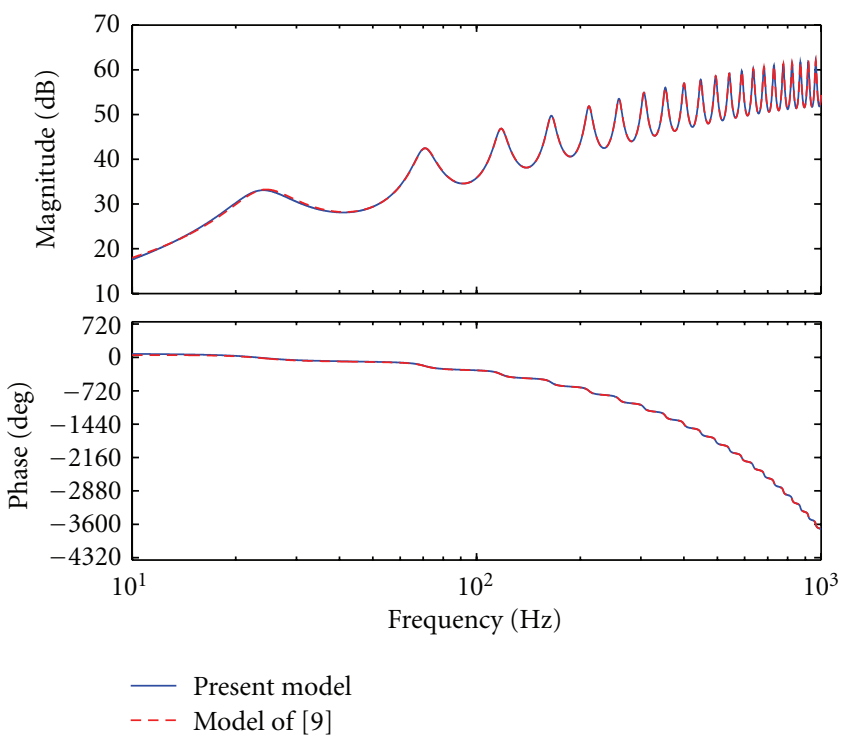

(a) Overview

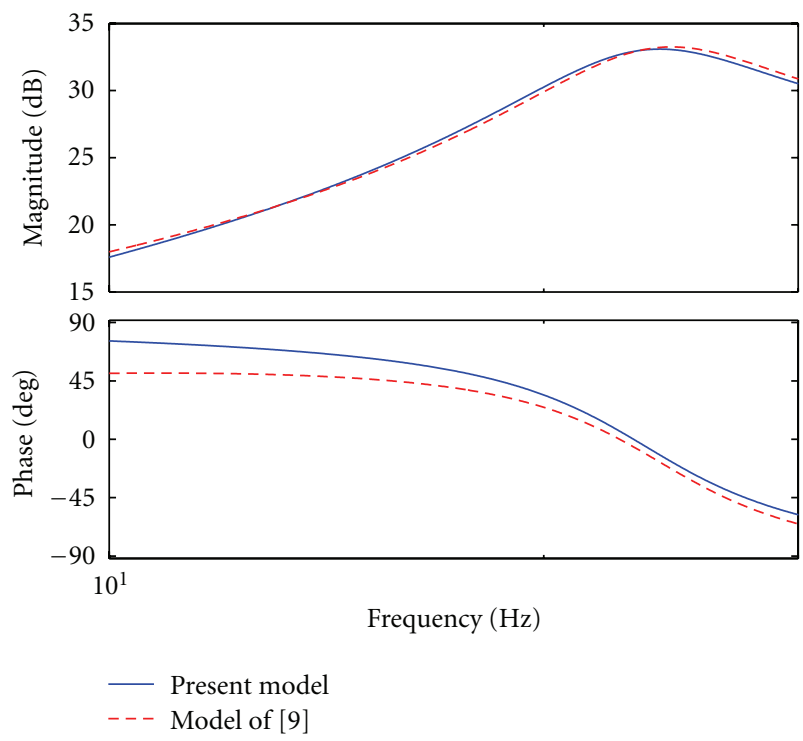

(b) Zoom on low frequencies

Figure 3: Comparison of $G_{z w}$ (Case A) with [9].

output $y$, and the error microphone output $z$ is used to evaluate control performance. Suppose that SPK1, Ref.Mic., SPK3, SPK2, and Err.Mic. are located at $x=0, l_{y}, l_{v}, l_{u}$, and $l_{z}$, respectively, in the main duct of length $L$.

There are three scenarios for the control source.

(i) Case A-Bidirectional source: SPK3 is not used; that is, $v(t)=0$ or $H=0$ and no subduct installed $\left(L_{S}=\right.$ $0)$. This can be described as bidirectional case since SPK2 emits waves in both directions.

(ii) Case B-Swinbanks' source: SPK3 is used to cancel the upstream sound produced by SPK2 without subduct $\left(L_{S}=0\right)$. It is, therefore, driven with the opposite signal, but delayed $v(t)=-u\left(t-\left(l_{u}-l_{v}\right) / c_{0}\right)$. This can be represented by the transfer function $H(s)=-e^{s\left(\left(l_{u}-l_{v}\right) / c_{0}\right)}$. (iii) Case C-rear sound aided source (proposed one): The rear sound of SPK2 is redirected by a subduct and attached in a way that the rear sound will interfere with the front sound at the junction of ducts. Note here that the assumption is made, that the rear sound travelling in the subduct can be simulated by a subduct of length $L_{S}=l_{u}-l_{v}$ with an additional loudspeaker (SPK3) to cover the rear sound. The amplitude of SPK3 can be adjusted by the variable $H$; that is, $u(t)=H \cdot v(t)$, where $H$ is treated as negative real number, whose magnitude is less than or equal to 1 , in this paper. ( $H$ is set to -1 when the strength of the rear sound is as the same to the front one.) The realizability of this setting is under consideration, however, one possibility of this is to cover the inside wall of the subduct by glass wool to weaken the rear sound. 
In Figure 2, $\left(p_{\bullet}, u_{\bullet}\right)$ represents the pressure and the particle velocity at each position in the duct. For the modelling process, the input signals $w, u$, and $v$ are assumed to be $u_{0}, u_{S}$ and $\tilde{u}_{S}$, respectively, neglecting the loudspeaker dynamics. The outputs $z$ and $y$ are assumed to be $p_{z}$ and $p_{y}$, respectively, neglecting the microphone dynamics. Thus, $u_{0}:=w, u_{S}:=u, \tilde{u}_{S}:=v, p_{z}:=z, p_{y}:=y$.
According to Lemma 2, the derivation process of the first principle model is eventually similar to the undamped case [9], where the derivation of the transfer function has been explained in detail. Hence, only the resultant transfer functions are shown as follows:

$$
\begin{aligned}
G_{0 u}= & \frac{Z_{0}\left(\sinh \left(\gamma\left(L-l_{u}\right)\right) \cosh \left(\gamma L_{s}\right)+\sinh \left(\gamma\left(L-l_{v}\right)\right) \cdot H\right)}{\sinh \left(\gamma\left(L-l_{v}\right)\right) \sinh \left(\gamma L_{s}\right) \cosh \left(\gamma l_{v}\right)+\cosh (\gamma L) \cosh \left(\gamma L_{s}\right)}, \\
G_{y u}= & G_{0 u} \cdot \cosh \left(\gamma l_{y}\right), \\
G_{z u}= & -Z_{0}\left(\sinh \left(\gamma\left(l_{z}-l_{u}\right)\right)+\frac{\sinh \left(\gamma\left(l_{z}-l_{v}\right)\right)}{\cosh \left(\gamma L_{s}\right)} \cdot H\right) \\
& +G_{0 u}\left(\sinh \left(\gamma\left(l_{z}-l_{v}\right)\right) \tanh \left(\gamma L_{s}\right) \cosh \left(\gamma l_{v}\right)+\cosh \left(\gamma l_{z}\right)\right), \\
& Z_{0}\left(\sinh \left(\gamma\left(L-l_{v}\right)\right) \tanh \left(\gamma L_{s}\right) \sinh \left(\gamma l_{v}\right)+\sinh (\gamma L)\right) \\
G_{0 w}= & \sinh \left(\gamma\left(L-l_{v}\right)\right) \tanh \left(\gamma L_{s}\right) \cosh \left(\gamma l_{v}\right)+\cosh (\gamma L) \\
G_{y w}= & \cosh \left(\gamma l_{y}\right) G_{0 w}-Z_{0} \sinh \left(\gamma l_{y}\right), \\
G_{z w}= & \left(\sinh \left(\gamma\left(l_{z}-l_{v}\right)\right) \tanh \left(\gamma L_{s}\right) \cosh \left(\gamma l_{v}\right)+\cosh \left(\gamma l_{z}\right)\right) G_{0 w} \\
& -Z_{0}\left(\sinh \left(\gamma\left(l_{z}-l_{v}\right)\right) \tanh \left(\gamma l_{s}\right) \sinh \left(\gamma l_{v}\right)+\sinh \left(\gamma l_{z}\right)\right) .
\end{aligned}
$$

It is obvious that for undamped case the derived functions and the ones given by [9] ought to be the same. This can be confirmed by setting the damping coefficient $d=0$ and using relationships between hyperbolic and trigonometric functions. Indeed, consistency has been confirmed for the undamped case. The major difference for the damped case is that the damping and acoustic impedance $Z_{0}$ considered in [9] are constant for all frequencies, while those in the derived functions above are frequency dependent: the imaginary part of the complex wave number in [9], $\alpha$ of $k=\left(\omega / c_{0}\right)-$ $j \alpha$, does not depend on the angular frequency $\omega$, while the one in the corresponding parameter $-j \gamma=\beta-j \alpha$ depends on $\omega$. Specifically, the difference mainly arises in low frequency range, since $\alpha$ given by (14) might be regarded as a constant number in high-frequency range, whose limit is given by $\lim _{\omega \rightarrow \infty} \alpha=d /\left(2 \rho_{0} c_{0}\right)$. Figure 3 shows the frequency response of Case A (only $G_{z w}$ is shown in the figure due to the limitation of the space) for the both considerations of damping, where the same parameters in [9] are used as follows:

$$
\begin{aligned}
& L=3.61, \quad l_{z}=3.53, \quad l_{u}=2.32, \quad l_{y}=0.03, \\
& c_{0}=340, \quad \rho_{0}=1.21 \text {. }
\end{aligned}
$$

The damping coefficient is set as $d=72.6$ which is obtained from the relationship $d /\left(2 \rho_{0} c_{0}\right)=30 / 340 \approx 0.09(0.09$ is given in [9]) so that both considerations of damping result similar damping effect in high-frequency range. Although overall bode characteristics did not change, some minor differences appear due to the different approach of taking damping into account in low frequencies as it is shown in Figure 3(b).

We here emphasize that the derived model in this paper is more relevant than that of [9], since the frequency response of the model can be directly compared with the one calculated by numerical implementation in the next section.

\section{Numerical Implementation}

The solution of the damped wave equation (3) using the transmission line theory shall be confirmed by comparing to an alternative approach, that is, numerical simulation by implementing a finite difference method, in which forward and backward difference are used to calculate the frequency response of the system.

In this section, the numerical algorithm for Case A will first be derived. Based on this, the numerical algorithm for Case $\mathrm{C}$ will be explained.

3.1. Case A. The effect of a loudspeaker in duct can be considered in the wave equation by using a spacial delta function [8]. Thus, the damped wave equation with a loudspeaker can be described as follows:

$$
\frac{1}{c_{0}^{2}} \frac{\partial^{2} p}{\partial t^{2}}+\frac{d}{\rho_{0} c_{0}^{2}} \frac{\partial p}{\partial t}=\frac{\partial^{2} p}{\partial x^{2}}+\rho_{0} \dot{u} \delta\left(x-l_{u}\right)
$$


For numerical analysis, time $t$ and space $x$ are discretized as follows:

$$
\begin{gathered}
t=t_{i}:=i \cdot \Delta t, \quad \forall i \in\{0,1, \ldots, N\}, \\
x=x_{j}:=j \cdot \Delta x, \quad \forall j \in\{0,1, \ldots, M\}, \\
N:=\frac{T}{\Delta t}, \quad M:=\frac{L}{\Delta x}, \quad j_{u}:=\frac{l_{u}}{\Delta x},
\end{gathered}
$$

where $T$ is a simulation period. After applying forward and backward difference on the damped wave equation (19), one obtains

$$
\begin{aligned}
& \frac{p\left(t_{i+1}, x_{j}\right)-2 p\left(t_{i}, x_{j}\right)+p\left(t_{i-1}, x_{j}\right)}{\Delta t^{2}} \\
& +\frac{d}{\rho_{0}} \frac{p\left(t_{i}, x_{j}\right)-p\left(t_{i-1}, x_{j}\right)}{\Delta t} \\
& \quad=c_{0}^{2} \frac{p\left(t_{i}, x_{j+1}\right)-2 p\left(t_{i}, x_{j}\right)+p\left(t_{i}, x_{j-1}\right)}{\Delta x^{2}}+c_{0}^{2} \rho_{0} \dot{u} \frac{\delta_{j j_{u}}}{\Delta x},
\end{aligned}
$$

where $\delta_{j j_{u}}$ denotes the Kronecker delta to approximate the original property of integration being 1 by summation as follows:

$$
1=\int_{0}^{L} \delta\left(x-l_{u}\right) d x \approx \sum_{j=0}^{M-1}\left(\frac{\delta_{j j_{u}}}{\Delta x}\right) \Delta x, \quad \delta_{i j}= \begin{cases}1, & (i=j), \\ 0 & (i \neq j) .\end{cases}
$$

Rearranging the recurrence relation for future time value and introducing auxiliary variables leads to

$$
\begin{aligned}
p\left(t_{i+1}, x_{j}\right)= & p\left(t_{i}, x_{j}\right) \cdot[2-s-2 r]+p\left(t_{i-1}, x_{j}\right) \cdot[s-1] \\
& +p\left(t_{i}, x_{j+1}\right) \cdot[r]+p\left(t_{i}, x_{j-1}\right) \cdot[r] \\
& +r \Delta x \rho_{0} \dot{u} \delta_{j j_{u}},
\end{aligned}
$$

$$
s=\Delta t \cdot \frac{d}{\rho_{0}}, \quad r=\left(\frac{c_{0} \cdot \Delta t}{\Delta x}\right)^{2} .
$$

Since the duct has two ends, boundary conditions have to be set accordingly. As it has been done in the analytical process, the pressure at the open end is set to zero; that is, $p\left(t_{i}, x_{M}\right)=$ 0 for all $i$. For the closed end at $x=0$, the pressure gradient has to be zero. Thus, the relation

$$
\begin{aligned}
& \left.\frac{\partial p(t, x)}{\partial x}\right|_{x=0} \\
& \quad \approx \frac{p\left(t_{i}, x_{0}\right)-p\left(t_{i}, x_{-1}\right)}{\Delta x}=0 \Longleftrightarrow p\left(t_{i}, x_{-1}\right)=p\left(t_{i}, x_{0}\right)
\end{aligned}
$$

TABLE 1: Numerical approximation settings.

\begin{tabular}{lccc}
\hline Parameter & Notation & Value & Unit \\
\hline Spatial step width & $\Delta x$ & 0.01 & $\mathrm{~m}$ \\
Simulation time & $T$ & 1 & $\mathrm{~s}$ \\
Time steps & $N$ & 40000 & \\
\hline
\end{tabular}

holds with a pressure at a fictitious location $x=-\Delta x=: x_{-1}$. This relation can be used in (23) for $j=0$ leading to

$$
\begin{aligned}
p\left(t_{i+1}, x_{0}\right)= & p\left(t_{i}, x_{0}\right) \cdot[2-s-r]+p\left(t_{i-1}, x_{0}\right) \cdot[s-1] \\
& +p\left(t_{i}, x_{1}\right) \cdot[r] .
\end{aligned}
$$

By using the relations above, frequency response of the feedback-path system $G_{y u}$ can be numerically calculated by injecting the source input as a sinusoidal signal $u\left(t_{i}\right)=$ $-U \cos \omega t_{i}$ :

(1) initial condition: $p\left(t_{0}, x_{j}\right)=p\left(t_{1}, x_{j}\right)=0$ for all $j$,

(2) for $i=1,2, \ldots, N-1$ repeat the following:

(a) at the open end: $p\left(t_{i}, x_{0}\right)=0$,

(b) at the closed end: (26),

(c) for $j=1,2, \ldots, M-1$ repeat (23). (only at the source position $j=j_{u}$, the last term $r \Delta x \rho_{0} \dot{u}\left(t_{i}\right) \delta_{j j_{u}}$ appears as $\left.r \Delta x \rho_{0} U \sin t_{i}\right)$.

For the stability of the numerical algorithm above, parameters in Table 1 are set. The steady state pressure variation at the microphone position $p\left(t_{i}, x_{j_{y}}\right)$ is evaluated to get gain and phase from the source input $u$ to the microphone output $y$, where $j_{y}$ is defined as $j_{y}:=l_{y} / \Delta x$. The other frequency responses for $G_{z u}, G_{z w}$, and $G_{y w}$ can be calculated in a similar way. For each transfer function 2000 logarithmically distributed frequencies have been simulated.

3.2. Case C. As Case $\mathrm{C}$ consists of the main duct and an additional subduct, the previous derivation has to be adapted accordingly. First, similar to the main duct, an additional $z$ axis is introduced so that SPK3 is located at $z=0$ and the subduct is connected at $z=L_{S}$ as shown in Figure 2, by which the instantaneous pressure in the subduct is denoted as $p_{S}(t, z)$. Then, $t$ and $z$ are discretized in a similar manner as Case $\mathrm{A}$

$$
z=z_{k}:=k \cdot \Delta x \quad \forall k \in\left\{0,1, \ldots, M_{S}\right\}, M_{S}:=\frac{L_{S}}{\Delta x}
$$

where common spacing $\Delta x$ is used. The input for SPK3 is specified as $v\left(t_{i}\right)=H u\left(t_{i}\right)$. The boundary condition for the closed end of the attached subduct is added like before. 


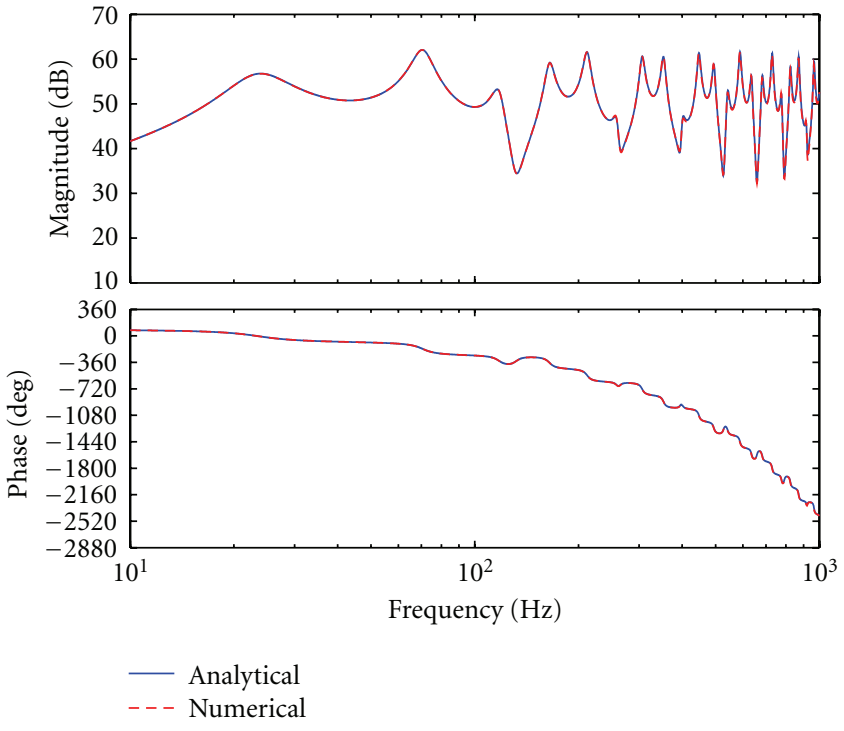

(a) Case $\mathrm{A}$

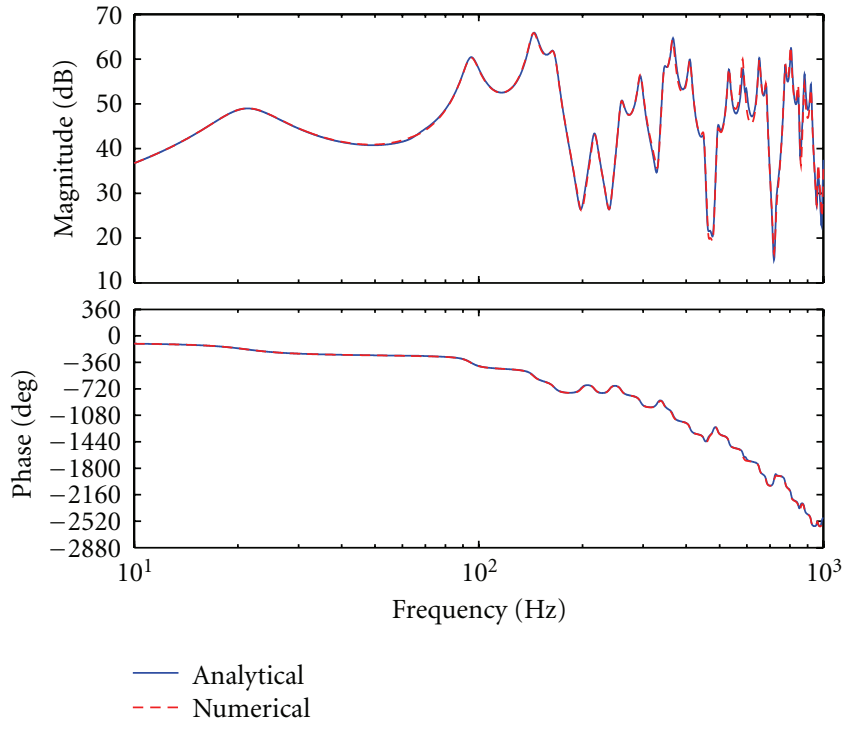

(b) Case C

FIgURE 4: Comparison of numerical and analytical solution, transfer function $G_{y u}, H=-1$.

Furthermore, the pressure at the junction is assumed as the average of all surrounding pressure points

$$
\begin{aligned}
& p\left(t_{i+1}, x_{j_{v}}\right) \\
& \quad=p_{S}\left(t_{i+1}, z_{M_{S}}\right) \\
& \quad=\frac{p\left(t_{i+1}, x_{j_{v}-1}\right)+p\left(t_{i+1}, x_{j_{v}+1}\right)+p_{S}\left(t_{i+1}, z_{M_{S}-1}\right)}{3},
\end{aligned}
$$

where $j_{v}:=l_{v} / \Delta x$. The relation above might now be only an assumption; however, the validity will be shown later. The numerical algorithm in detail is omitted from the paper, since it is parallel to Case A based on the additional setting for Case C. So far, two solutions of the frequency response based on the damped wave equation (3) have been obtained. In the remaining of this section, the results will be compared. Figure 4 shows frequency response of the feedback-path system for Cases A and C.

Apparently, both solutions match almost perfect as it can be seen in above figures. This testifies the application of transmission line theory and numerical simulation via finite difference method with the applied assumptions. However, the desired additional phase lag of Case $\mathrm{C}$ compared to Case A did not occur. In both cases, the total phase lag is about 2520 degree. A possible cause for the missing phase lag could be a different setting of the rear-sound strength $H$, which leads to different bode diagrams for $G_{y u}$ as pointed in [9].

\section{Optimal Condition for the Longest Time Delay in Feedback-Path System}

In order to examine the effect of a different setting of the rear-sound strength $H$, the analytic solution is modified and

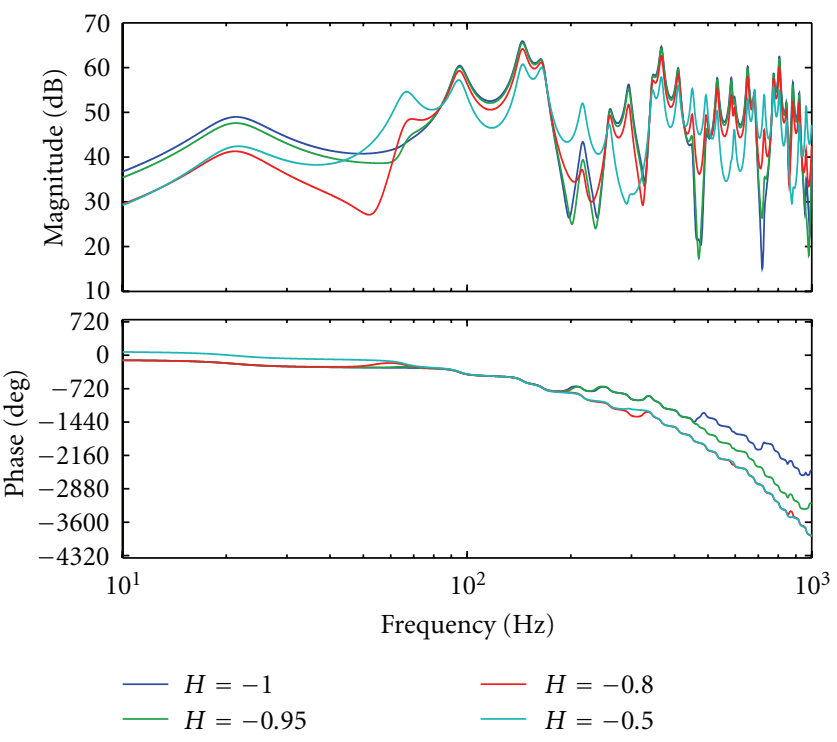

FIGURE 5: Comparison of transfer function $G_{y u}$ of Case C for different rear-sound strengths $H$.

compared to itself with several settings for $H$, as it can be seen in Figure 5.

Evidently, a lower value of $H$ will lead to the desired effect and, thus, shows an advantage over the bidirectional scenario. The reason for that behaviour can be found in the transfer function for $G_{0 u}$, since it is part of $G_{y u}$. For the explanation, the undamped equation will be used as it simplifies the next steps and did not show much influence on phase characteristics. The derivation will be shown for the transfer function of Case A, the same method can later be applied to Case C. Firstly, $G_{0 u}$ will be simplified for Case A by 


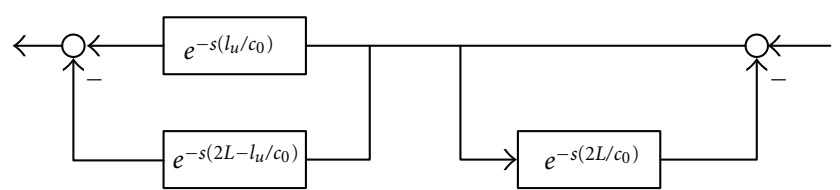

FIGURE 6: Signal flow chart in frequency domain.

setting $L_{s}=0, H=0$ and rewriting as exponential functions. After making the equation causal by multiplying with the longest time delay $e^{-j k L}$ to both denominator and numerator, and replacing $j k$ by $s / c_{0}$, the following is obtained:

$$
G_{0 u}=\rho_{0} c_{0} \frac{e^{-s\left(l_{u} / c_{0}\right)}-e^{-s\left(2 L-l_{u}\right) / c_{0}}}{1+e^{-s\left(2 L / c_{0}\right)}} .
$$

This transfer function corresponds with a causal system depicted in Figure 6, where each signal block represents a time delay.

It can be observed that the shortest time delay is given by $e^{-s\left(l_{u} / c_{0}\right)}$. As mentioned in the introduction, the value of the shortest time delay is critical for the control performance. Similar for Case C, the same procedure is applied and some variables for better overview are introduced. Since the denominator is not of relevance and long, it has been neglected here and the modified transfer function $G_{0 u}$ can be derived as

$$
\begin{aligned}
G_{0 u}=4 \rho_{0} c_{0}( & \left(e^{-s\left(3 l_{u}-2 l_{v}\right) / c_{0}}-e^{-s\left(2 L-l_{u}\right) / c_{0}}\right) \\
& \left.+[(1 / 2)+H]\left(e^{-s\left(l_{u} / c_{0}\right)}-e^{-s\left(2 L-2 l_{v}+l_{u}\right) / c_{0}}\right)\right) .
\end{aligned}
$$

From this equation, it becomes clear that half rear-sound amplitude will eliminate the shortest time delay given by $e^{-s\left(l_{u} / c_{0}\right)}$ and leads to $e^{-s\left(3 l_{u}-2 l_{v}\right) / c_{0}}$ as the shortest time delay. Compared to the previous result for Case A (29), an additional time delay of $e^{-s\left(2 l_{u}-2 l_{v}\right) / c_{0}}$ could be achieved. This corresponds to twice the length of the subduct and can be seen in Figure 5. The time delay can also be verified by multiplying the transfer function $G_{y u}$ of Case A by an additional, fictitious time delay. In Figure 7, it can be seen that this modified Case A and Case $\mathrm{C}$ with a rear-sound strength of -0.5 produce indeed a very similar phase lag.

The numerical implementation reveals the additional time delay in feedback-path system by displaying the propagation of the incident waves. In Figures 8 and 9, the wave propagation for Case A and Case $\mathrm{C}$ is shown. The $y$-axis depicts the acoustic pressure distribution for different points in time, the $x$-axis represents the position in the main duct, where the closed end is located at $x=0$. One complete period with a frequency of $1000 \mathrm{~Hz}$ is excited by the control loudspeaker at $l_{u}=2.32$, and the propagation through the duct is observed. Note that the first upwards travelling wave is extinguished by the rear sound in Case $\mathrm{C}$ and previously derived time delay is obtained.

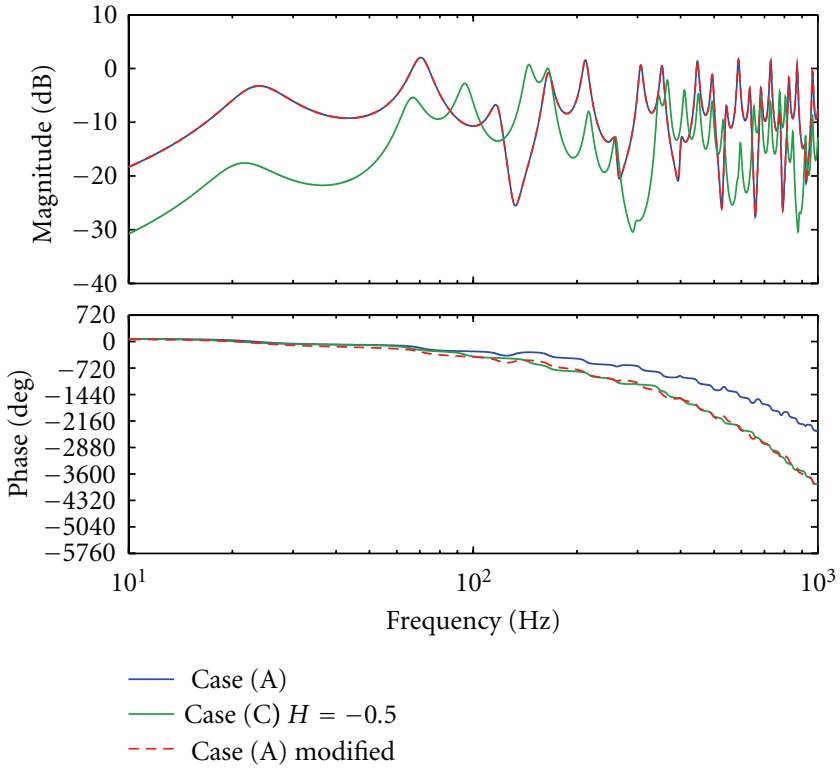

Figure 7: Comparison of phase characteristics for Case A, Case A with additional time delay, and Case $\mathrm{C}(H=-0.5)$.

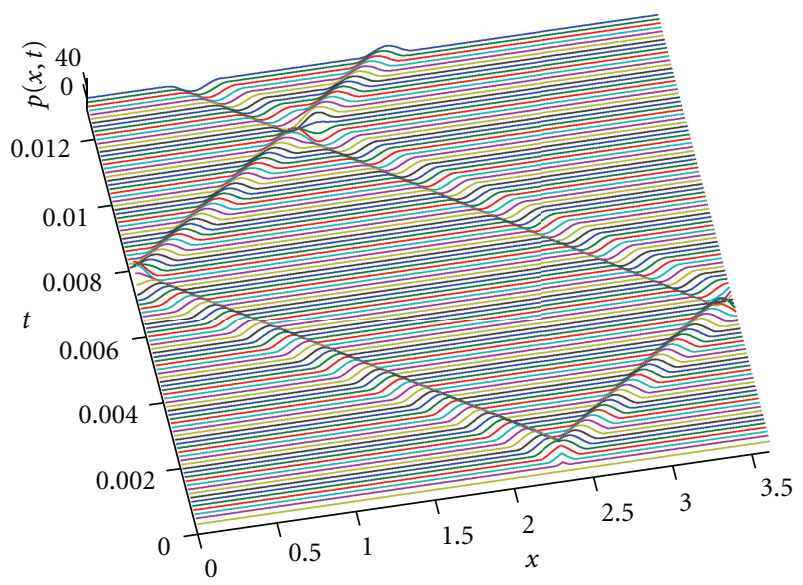

Figure 8: Numeric time response, Case A.

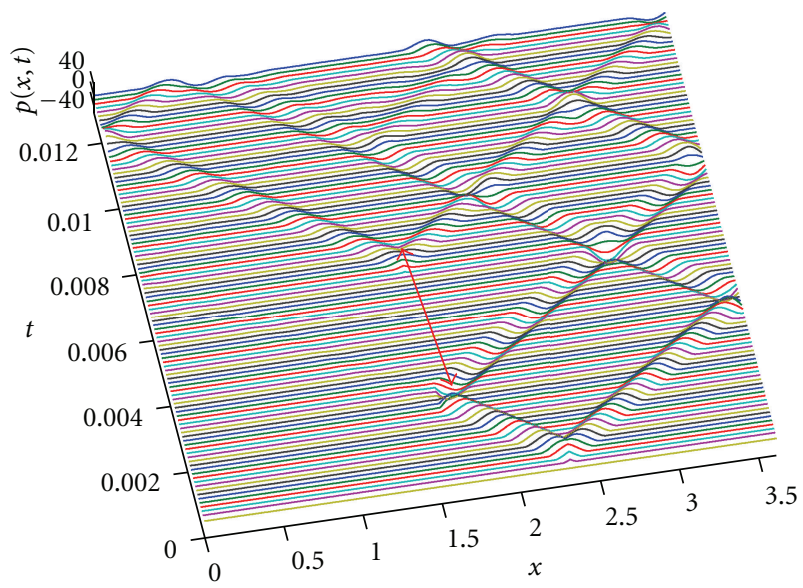

Figure 9: Numeric time response, Case C. 


\section{Conclusion}

In this paper, as an additional approach in active noise control next to the Swinbanks' source, the proposed rearsound-aided source has been analytically examined. The advantage of additional phase lag and time delay in the feedback-path transfer function of the proposed control source has been analyzed. To accomplish this, the damped wave equation has been used and based on this: a first principle model has been derived by the application of transmission line theory. The model has been confirmed by verifying that

(i) the previous model is included as the special case of an undamped scenario,

(ii) the frequency response is consistent with the one obtained by a numerical simulation based on a finite difference implementation of the damped wave equation.

It has been shown, that the different consideration of damping induced some minor changes in low-frequency characteristics. Characteristics in middle and high-frequency range were consistent to the previous model, which implies validity of the previous results in [9]: The proposed control source has shown additional phase lag by assuming a weaker rear sound. The advantage has also been examined from a theoretical point of view in order to determine under which conditions an additional time delay is achieved. It has been demonstrated that the shortest time delay in the feedback-path transfer function is extended by $2 L_{S} / c_{0}$, when $H=-0.5$, where $L_{S}$ resembles the length of the subduct and $c_{0}$ the speed of sound. This time delay has been also confirmed by comparison with the phase characteristic for the bidirectional source with additional time delay. In addition, the time delay has been directly confirmed in the time response simulation of the pressure variation by an incident wave.

The presented results have analytically proved that the proposed control source induces additional time delay in the feedback-path transfer function as compared to the conventional bidirectional source by a suitable choice of $H$. This ends up showing that the original setting of $H=-1$ is not suitable for the proposed source to maximize the additional time delay, which might be inconsistent with the result in [9], since the similar phase characteristic of Swinbanks' source has been achieved by $H=-1$ in the experiment. To tackle this problem, the time response simulation in this paper will be used for comparison in detail with a measured time response by experiments. Further effect of the rear-sound amplitude on control performance will be examined.

\section{References}

[1] M. A. Swinbanks, "The active control of sound propagating in long ducts," Journal of Sound and Vibration, vol. 27, pp. 411436, 1973.

[2] S. Kijimoto, H. Tanaka, Y. Kanemitsu, and K. Matsuda, "Howling cancellation for active noise control with two sound sources," Transactions of the Japan Society of Mechanical Engineers. C, vol. 67, no. 656, pp. 52-57, 2001 (Japanese).

[3] Y. Kobayashi and H. Fujioka, "Active noise cancellation for ventilation ducts using a pair of loudspeakers by sampled-data $\mathfrak{H} \infty$ optimization," Advances in Acoustics and Vibration, vol. 2008, Article ID 253948, 8 pages, 2008.

[4] J. Winkler and S. J. Elliott, "Adaptive control of broadband sound in ducts using a pair of loudspeakers," Acustica, vol. 81, no. 5, pp. 475-488, 1995.

[5] Y. Kobayashi and H. Fujioka, "Analysis for robust active noise control systems of ducts with a pair of loudspeakers," in Proceedings of the International Congress on Sound and Vibration (ICSV'08), Daejeon, Korea, 2008.

[6] Y. Kobayashi and H. Fujioka, "Robust stability analysis for active noise control systems of ducts with a pair of loudspeakers," in Proceedings of the 37th Symposium on Control Theory (SICE '08), pp. 21-247, 2008.

[7] D. S. Bernstein, "What makes some control problems hard?" IEEE Control Systems Magazine, vol. 22, no. 4, pp. 8-19, 2002.

[8] J. Hong and D. S. Bernstein, "Bode integral constraints, colocation, and spillover in active noise and vibration control," IEEE Transactions on Control Systems Technology, vol. 6, no. 1, pp. 111-120, 1998.

[9] Y. Kobayashi, H. Fujioka, and N. Jinbo, "A control source structure of single loudspeaker and rear sound interference for inexpensive active noise control," Advances in Acoustics and Vibration, vol. 2010, Article ID 730813, 9 pages, 2010.

[10] W. Barten, A. Manera, and R. Macian-Juan, "One- and twodimensional standing pressure waves and one-dimensional travelling pulses using the US-NRC nuclear systems analysis code TRACE," Nuclear Engineering and Design, vol. 238, no. 10, pp. 2568-2582, 2008.

[11] P. A. Nelson and S. J. Elliott, Active Control of Sound, Academic Press, New York, NY, USA, 1993.

[12] S. J. Elliott, Signal Processing for Active Control, Volume in the Signal Processing and Its Applications Series, Academic Press, New York, NY, USA, 2009. 

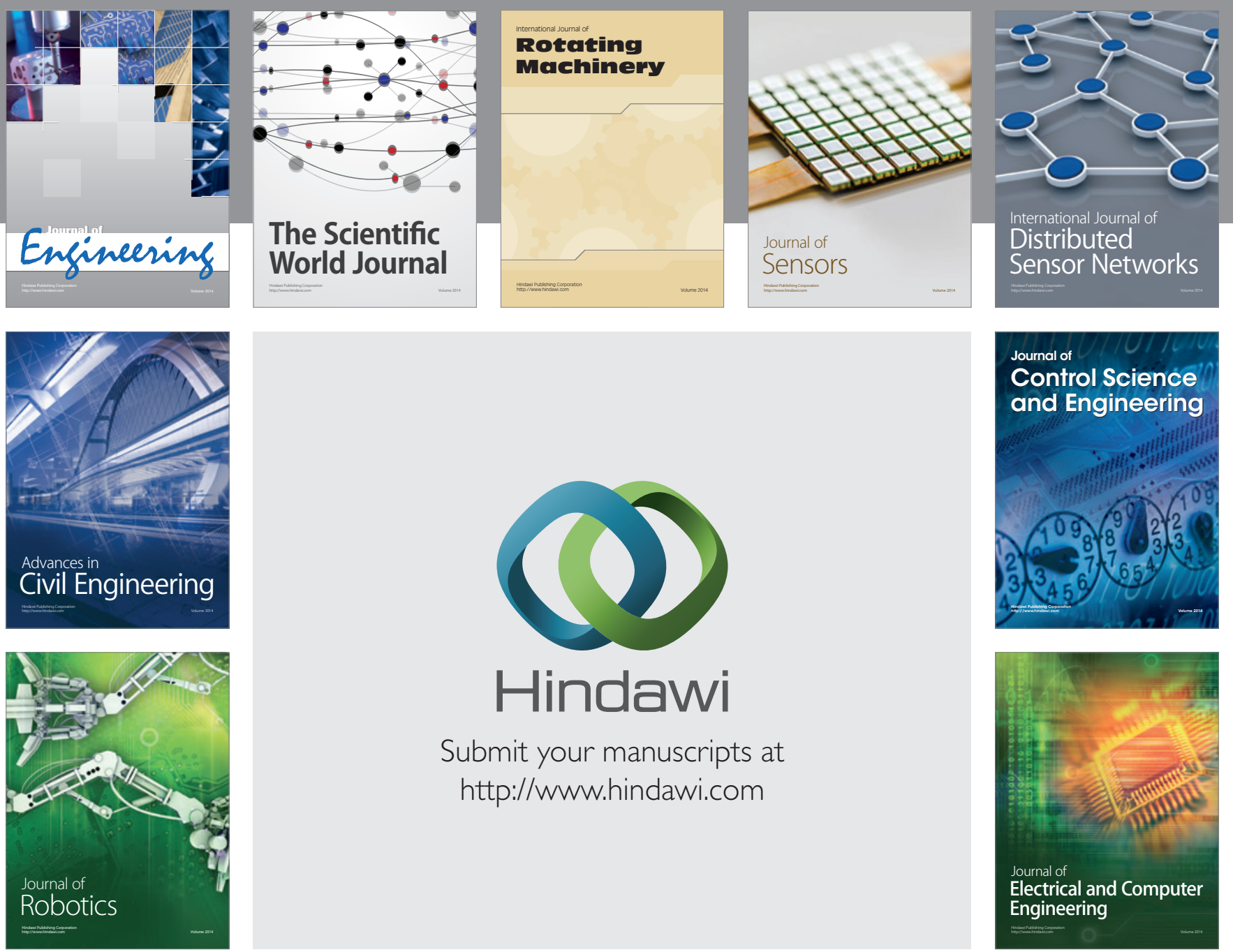

Submit your manuscripts at

http://www.hindawi.com
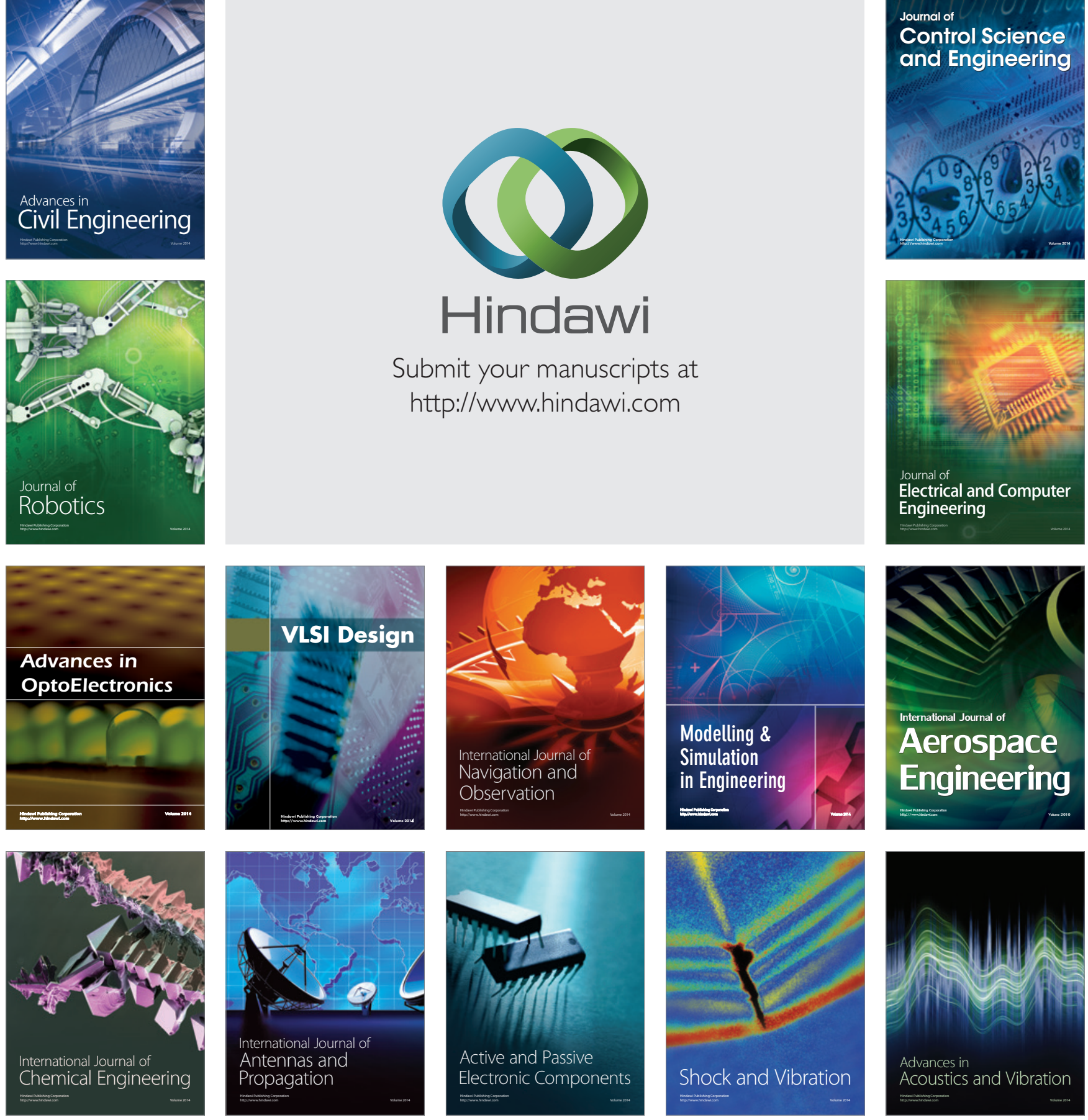